\title{
EVALUACIÓN DE UN ESTUDIANTE CON TDAH TRAS UNA INTERVENCIÓN CON RECURSOS TECNOLÓGICOS
}

\author{
Assessment of a Student with ADHD \\ after an Intervention with Technological Resources
}

\author{
Isabel Fernández-Menor ${ }^{1}$ \\ ORCID: 0000-0003-2873-6952 \\ 1 Universidad de Vigo, España \\ Correspondencia: isfernandez@uvigo.es
}

Recibido: $27 / 04 / 2021$

Aceptado: 16/09/2021

Resumen: Esta investigación analiza la importancia de las intervenciones realizadas con tecnologías de información y comunicación (TIC) o tecnologías de apoyo a la diversidad (TAD) en la mejora de alumnado con necesidades específicas de apoyo educativo (NEAE). Así, se realiza una intervención con diferentes tipologías de software educativo para mejorar la atención, la memoria, la concentración, la organización, la relajación y las habilidades sociales de un alumno con TDAH de doce años. Para ello, se ha diseñado una intervención basada en un estudio de caso único de tipo $\mathrm{A}_{1}-\mathrm{B}-\mathrm{A}_{2}$ y paradigma mixto, durante diecinueve sesiones. Los instrumentos de recogida de información han sido D.N: CAS, d2, MFF-20, diario de campo y escala de observación. Asimismo, la intervención utiliza diferentes tipologías de software y dispositivos tecnológicos para trabajar los aspectos antes citados. Los resultados muestran la mejora en todas las áreas, a excepción de la memoria.

Palabras clave: TDAH; TIC; intervención educativa; software educativo; estudio de caso.

\begin{abstract}
This research analyzes the importance of interventions carried out with information and communication technologies (ICT) or diversity support technologies in the improvement of students with specific educational support needs. Thus, an intervention is carried out with different types of educational software to improve attention, memory, concentration, organization, relaxation and social skills of a twelve-year-old student with ADHD. For this purpose, an intervention has been designed based on a single case study of $A_{l}-B-A_{2}$ type and mixed paradigm, during nineteen sessions. The data collection instruments were D.N: CAS, d2, MFF-20, field diary and observation scale. Likewise, the intervention used different types of software and technological devices to work on the mentioned aspects. The results show improvement in all areas, except for memory.
\end{abstract}

Keywords: ADHD; ICT; educational intervention; educational software; case study. 


\section{Introducción}

En algún momento de su vida docente, cualquier maestro o maestra se ha encontrado con estudiantes con dificultades para mantener la atención, con una excesiva impulsividad, bajo autocontrol o problemas para planificar, organizar o revisar. Estas características, las cuales afectan a su rendimiento, funciones ejecutivas, autoestima y relaciones con los demás, describen al alumnado con trastorno por déficit de atención e hiperactividad (TDAH).

El TDAH es el trastorno más frecuente del neurodesarrollo (Encalada, 2019; López et al., 2020; Rodillo, 2015) y, según los datos ofrecidos por la Organización Mundial de la Salud (OMS), es padecido por un 5,3\% de la población (Alonso, 2020). Aunque las causas todavía no están definidas, las investigaciones se inclinan hacia un origen neurobiológico con una importante vinculación genética, así como la interacción con diversos factores ambientales tales como la prematuridad o el consumo de alcohol y tabaco durante el embarazo. Con estas referencias, se pueden atisbar las dificultades que, a nivel educativo, supone para estos estudiantes la presencia del trastorno.

Entre las medidas educativas más recurridas están el desarrollar la atención, el autocontrol, ofrecer instrucciones individualizadas, tener en cuenta su ritmo de aprendizaje u ofrecer tareas con un carácter más práctico (Rabadán et al., 2017). Estas tareas ordinarias pueden desarrollarse, también, a través de las tecnologías de la información y la comunicación (TIC), por ser herramientas funcionales en la atención a la diversidad, concretamente por su versatilidad, motivación o fomento de la individualización de la enseñanza.

El objetivo general de este estudio es conocer y analizar los efectos de la intervención con software educativo en la intervención de un sujeto de doce años con TDAH que presenta dificultades en atención, memoria, concentración, organización, relajación y habilidades sociales.

\section{EI empleo de las TIC en el trabajo con estudiantes con TDAH}

El alumnado con TDAH está en riesgo en cualquier sistema educativo a nivel mundial, debido a que no responde al patrón establecido. Su impaciencia, falta de atención, problemas de memoria, impulsividad, exceso de movimiento o comportamientos disruptivos (Gil y Megías, 2017; Raposo y Salgado, 2015) alteran el orden del aula preocupando a docentes y otros profesionales del ámbito educativo. No obstante, la atención a la diversidad ha de ser 
garantizada para todos y cada uno de los estudiantes. En este marco, las TIC permiten a las personas con necesidades específicas de apoyo educativo (NEAE) acceder a los objetivos y contenidos con una mayor variedad de canales, respetando el ritmo de aprendizaje, los que resultan más motivadores (Chousa et al., 2017). Concretamente, cuando se habla de TIC aplicadas a la atención de personas con NEAE se hace referencia a las TAD (tecnologías de apoyo a la diversidad), que se definen como "cualquier producto (incluidos dispositivos, equipo, instrumentos, tecnologías y software) fabricado especialmente o disponible en el mercado para prevenir, compensar, controlar, mitigar o neutralizar deficiencias, limitaciones de la actividad o restricciones en la participación” (Doval et al., 2013, p. 14).

El uso de la tecnología en el TDAH puede ser empleado de tres formas diferentes, tal y como indican González y Oliver (2002). En primer lugar, para evaluar, no solo diagnósticamente, sino también su proceso de aprendizaje. En segundo lugar, como reforzador secundario, favoreciendo la resolución de actividades. En tercer y último lugar, como regulador de la atención, mejorando el control de la conducta. Teniendo en cuenta las bondades de las tecnologías en la aplicación al trabajo con alumnado con TDAH, es preciso indicar algunos criterios que la tecnología ha de seguir: ha de ser motivadora (atractiva a la atención en cuanto a diseño, gráficas, música o con personajes como hilo conductor), con tareas lúdicas (evitando la monotonía), sin excesivas animaciones, que destaque los aciertos y disimule los errores (con motivo de mejorar la autoestima y evitar la frustración al fracaso), con un grado de dificultad asequible, actividades que favorezcan la tranquilidad, con verbalizaciones guiadas y con autoevaluación reforzada (Campos et al., 2017; Chousa, et al., 2017).

A pesar de que existen investigaciones relevantes que vinculan el TDAH con elementos como la memoria — por ejemplo, el estudio de Capodieci et al. (2019) o el de Crisol y Campos (2019) — , lo cierto es que la investigación no es muy amplia en lo que a TIC y TDAH se refiere. Sin embargo, se pueden destacar estudios importantes en el campo. Uno de ellos es el de Álvarez Higuita (2009), quien llega a la conclusión de que la estructura narrativa de los estudiantes con TDAH no se ve condicionada por la herramienta con la que escriben. Otra investigación es la de Marco (2010), quien emplea el programa MeMotiva, también utilizado en este estudio, para mejorar la impulsividad, memoria operativa, atención sostenida y focalizada, comprensión lectora y tareas cognitivas como cálculo, capacidad organizativa y planificación. 


\section{Método}

Este estudio presenta los resultados de una investigación que emplea recursos tecnológicos en la intervención con un estudiante con TDAH y problemas con la atención, la memoria, la concentración, la organización, la relajación y las habilidades sociales. Para ello, se ha diseñado una investigación de tipo $\mathrm{A}_{1}-\mathrm{B}-\mathrm{A}_{2}$. Este diseño consta de una fase $\mathrm{A}_{1}$, denominada pretest, correspondiente con las dos primeras sesiones de intervención en la cual se realizan las valoraciones iniciales del alumno. Estas dos primeras sesiones tienen una duración de 55 minutos cada una y se han realizado en el mes de enero del año 2019. La fase $\mathrm{B}$, de intervención, se desarrolla en 15 sesiones de 55 minutos cada una. Se han llevado a cabo entre los meses de enero y abril del año 2019. El software empleado se expone en el apartado metodológico. Finalmente, la fase $\mathrm{A}_{2}$, denominada postest, se realiza en el mes de mayo de 2019 y responde a las mismas características mencionadas en la primera de las fases $\left(\mathrm{A}_{1}\right)$ para valorar la existencia o no de diferencias en los resultados, tras la intervención.

\section{Muestra}

Esta investigación consta de un único participante de doce años de edad y que cursa sexto de Educación Primaria, por segunda vez, en un colegio público de Ourense (España). En lo relativo a su historial académico el alumno presenta, según sus docentes, un rendimiento bajo en todas las lenguas, ciencias sociales y ciencias de la naturaleza; no obstante, destaca en matemáticas o educación física. Su bajo rendimiento puede deberse a sus dificultades atencionales. El alumno acude a un aula con un profesor especialista una hora cada día, en la cual se llevan a cabo tareas de refuerzo educativo.

\section{Instrumentos}

Los instrumentos empleados para la evaluación pretest y postest del estudiante son tres: el Sistema de Evaluación Cognitiva D.N: CAS (Deaño, 2007), el test d2 (Brickenkamp y Cubero, 2002) y el test de emparejamiento de figuras conocidas MFF-20 (Buela et al., 2005). El primero de ellos evalúa procesos cognitivos como planificación, atención, procesamiento simultáneo y sucesivo. El segundo, valora la atención sostenida y la velocidad de procesamiento. El tercero mide la reflexividad e impulsividad frente a una tarea. 
Durante la intervención se han empleado los recursos mostrados en la Tabla 1. Con motivo de anotar comportamientos y conductas, y valorar la actitud general del sujeto en la sesión, la utilidad del dispositivo tecnológico y la utilidad del software, se han utilizado el diario de campo y tres escalas de observación.

Tabla 1

Categoría, nombre y área que trabajan los instrumentos de la intervención

\begin{tabular}{lll}
\hline Categoría & Nombre de los recursos & Área de trabajo \\
\hline $\begin{array}{l}\text { Aplicaciones para móvil y } \\
\text { tableta }\end{array}$ & $\begin{array}{l}\text { Siluetas OA, Pares, Tetris, } \\
\text { Simon dice, Touch the } \\
\text { numbers, } \\
\text { proyect@ habilidades, } \\
\text { Memorando, NeuroNation, } \\
\text { Memory Trainer y TDAH }\end{array}$ & $\begin{array}{l}\text { Atención, memoria, } \\
\text { concentración, habilidades } \\
\text { sociales, relajación y } \\
\text { organización }\end{array}$ \\
& $\begin{array}{l}\text { Trainer } \\
\text { Word y Childsplay }\end{array}$ & $\begin{array}{l}\text { Organización, memoria, } \\
\text { atención y concentración } \\
\text { Memoria, atención, } \\
\text { Programas informáticos }\end{array}$ \\
Páginas web & $\begin{array}{l}\text { Clic,Clic,Clic, Genmagic e } \\
\text { Hypatiamat }\end{array}$ & $\begin{array}{l}\text { y relajación } \\
\text { Habilidades sociales, } \\
\text { memoria y concentración }\end{array}$ \\
Videojuego & La isla del tesoro & $\begin{array}{l}\text { Habilidades sociales } \\
\text { Memoria }\end{array}$ \\
\hline
\end{tabular}

Fuente: Elaboración propia (2021)

\section{Resultados}

En este apartado se presentan los resultados obtenidos antes y después de la aplicación de la intervención con recursos tecnológicos con el estudiante con TDAH. Posteriormente se evalúa la actitud del alumno durante las sesiones, la utilidad del dispositivo y la utilidad del recurso tecnológico en cuanto a su función metodológica y su adecuación a las características de este trastorno.

A través del D.N. CAS se ha medido la atención y la memoria, mediante el test d2 se ha medido la atención y la concentración y, finalmente, con el test MFF-20 se observa la variación en la impulsividad frente a la reflexión en la tarea, es decir, la relajación del alumno ante la actividad. Los resultados pre y postest obtenidos en estas pruebas se observan en la Tabla 2. 
Tabla 2

Puntuaciones obtenidas previa y posteriormente a la aplicación de la intervención

\begin{tabular}{lll}
\hline Prueba & Pretest & Postest \\
\hline D.N: CAS & 100 & 128 \\
d2 & 80 & 99 \\
MFF-20 & Impulsividad: 90 & Impulsividad: 40 \\
& Ineficacia: 40 & Ineficacia: 1 \\
\hline
\end{tabular}

Fuente: Elaboración propia (2021)

Los procesos medidos a través del D.N: CAS muestran un incremento significativo en la escala completa tras la intervención, de 100 a 128 puntos. En las pruebas que incluyen esta escala completa (planificación, simultáneo, sucesivo y atención), todas han experimentado un incremento significativo, a excepción del procesamiento sucesivo que se mantiene igual.

En cuanto al test d2, las puntuaciones obtenidas en la primera aplicación eran positivas, no obstante, el estudiante presentaba puntuaciones preocupantes en omisiones y comisiones, lo que indicaba un problema en su atención sostenida. En la segunda aplicación, todas las puntuaciones mejoran considerablemente y el sujeto ya no marca estímulos incorrectos (comisiones), aunque sigue presentando un pequeño número de omisiones, lo que significa que deja estímulos sin marcar a lo largo de la tarea.

Con relación al test de emparejamiento de figuras conocidas MFF-20, en la fase de pretest, la impulsividad ante la tarea era muy elevada (90), a pesar de que su efectividad se encontraba cerca de la media (40). En este sentido, antes de la intervención, el alumno se podía categorizar como un impulsivo eficiente. Sin embargo, tras la intervención, su impulsividad descendió hasta encontrarse en un punto medio entre impulsividad y reflexividad (40) y, además, su eficiencia mejoró notablemente, alcanzando el máximo posible (1). En esta línea, se puede decir que la impulsividad del alumno se redujo a favor de una mayor reflexividad.

Si vemos estos resultados, en función de las variables que se pretenden medir (ver Tabla 3), se observa una mejora en todas ellas a excepción de la memoria. 
Tabla 3

Puntuaciones pretest y postest en función a las variables a evaluación

\begin{tabular}{|c|c|c|c|}
\hline Variable & Tarea & Pretest & Postest \\
\hline \multirow[t]{3}{*}{ Memoria } & $\begin{array}{l}\text { Series de palabras (D.N: } \\
\text { CAS) }\end{array}$ & 10 & 8 \\
\hline & $\begin{array}{l}\text { Repetición de frases } \\
\text { (D.N: CAS) }\end{array}$ & 7 & 7 \\
\hline & $\begin{array}{l}\text { Preguntas sobre frases } \\
\text { (D.N: CAS) }\end{array}$ & 6 & 8 \\
\hline Concentración & Concentración (d2) & 75 & 98 \\
\hline \multirow[t]{5}{*}{ Atención } & Atención (d2) & 80 & 99 \\
\hline & $\begin{array}{l}\text { Atención expresiva } \\
\text { (D.N: CAS) }\end{array}$ & 6 & 10 \\
\hline & $\begin{array}{l}\text { Búsqueda de números } \\
\text { (D.N: CAS) }\end{array}$ & 8 & 14 \\
\hline & $\begin{array}{l}\text { Atención receptiva (D.N: } \\
\text { CAS) }\end{array}$ & 11 & 15 \\
\hline & Atención (D.N: CAS) & 25 & 39 \\
\hline Relajación & $\begin{array}{l}\text { Impulsividad/ineficiencia } \\
\text { (MFF-20) }\end{array}$ & $90 / 40$ & $40 / 1$ \\
\hline
\end{tabular}

Fuente: Elaboración propia (2021)

A través del diario de campo se ha recabado información sobre la organización y las habilidades sociales del alumno (trae el material que se le pide, mantiene el lugar de trabajo ordenado, sigue un orden en la realización de las tareas o mejora sus habilidades sociales), observándose una mejora en lo referente a estas variables. De esta manera, es importante destacar el uso de una agenda y el establecimiento de esta como un hábito en su vida académica, mejorando notablemente en su organización. Además, las notas recogidas en el diario evidencian la mejora de sus habilidades sociales.

A partir de la escala de observación se ha tratado de valorar la actitud general del sujeto en las sesiones, cuyos resultados han sido: no muestra dificultades para seguir instrucciones, no evita iniciar la tarea, no se levanta en situaciones en las que se espera que permanezca sentado, no habla de forma excesiva o cuando se espera que permanezca callado, no muestra demasiadas 
dificultades para prestar atención, suele escuchar cuando se le habla y no se distrae fácilmente una vez concentrado en la tarea. Sin embargo, si la tarea le resulta difícil o muy prolongada en el tiempo tiene dificultades para mantenerla y terminarla justificando este hecho con interjecciones de incapacidad. También tiende a olvidar rápidamente lo que ha realizado, sobre todo, en aquellas tareas que le han causado menos impacto. Además, responde impulsivamente antes de haber finalizado la pregunta o explicación, adelantándose así a la ejecución.

Por otro lado, con motivo de valorar la utilidad y experimentar diferentes tipologías de software educativo en la intervención de este trastorno, se ha empleado una escala de observación donde se valora la utilidad del dispositivo tecnológico empleado (móvil, ordenador o tableta) y el software utilizado.

En cuanto a la utilidad del dispositivo tecnológico empleado, se ha observado que móvil, ordenador y tableta son igualmente útiles a hora de controlar y ajustar la tarea, resolver problemas y adaptar los ritmos de aprendizaje. Se destaca que móvil y tableta resultan más motivadores y ofrecen una mayor variedad sensorial para el alumno que el ordenador.

Con relación a la utilidad del software empleado y en lo referente a su función metodológica, todas las tipologías de software - aplicaciones, software específico, actividades creadas con herramientas de autor, páginas web, videojuego y programas informáticosofrecen una responsabilidad total de la tarea y permiten generalizar los procesos. El videojuego o el uso de páginas web puede ser empleado como una recompensa para el estudiante. Las aplicaciones, el software específico y las páginas web permiten una mayor secuenciación de la tarea que las otras herramientas.

En cuanto a la utilidad del software empleado y su pertinencia en el empleo con estudiantes con TDAH, se puede destacar que los hay más y menos adecuados al uso con alumnado con problemas atencionales. En primer lugar, los programas informáticos resultan ser los menos motivadores para el alumno, mientras que las aplicaciones y el videojuego lo son más. Con relación a la evitación de las animaciones excesivas, el software específico y las actividades desarrolladas con herramientas de autor son más pertinentes, mientras que las aplicaciones lo son menos. En lo que respecta a la evitación de la frustración ante el error, todas las herramientas son adecuadas. El videojuego y el software específico ofrecen mejores verbalizaciones guiadas, mientras que en el caso de los programas informáticos son inexistentes. Con respecto al fomento de la tranquilidad, el software específico y programas informáticos permiten en mayor medida este hecho, resultando las aplicaciones, las actividades 
desarrolladas con herramientas de autor y el videojuego los menos facilitadores de la tranquilidad, sobre todo, por el trabajo contrarreloj. Finalmente, las aplicaciones y el videojuego ofrecen los entornos más lúdicos.

\section{Conclusiones}

El objetivo general de este estudio se ha cumplido parcialmente, ya que la memoria, correspondiente al procesamiento sucesivo, se mantiene igual. En este sentido, se puede valorar positivamente este objetivo, a pesar de que uno de los elementos no se haya alcanzado, probablemente por la necesidad de entrenamiento de esta área en un tiempo más prolongado y constante. La atención, medida por el D.N: CAS (Deaño, 2007) y por el test d2 (Brickenkamp y Cubero, 2002), mejora notablemente y experimenta un cambio significativo. La concentración, medida por el test d2, también experimenta un importante incremento. La relajación, medida por el test MFF-20 (Buela et al., 2005), muestra el cambio del alumno desde un sujeto impulsivo-eficiente en la tarea, hasta su situación en la media entre la impulsividad y la reflexividad siendo muy eficiente en la tarea. Finalmente, la organización y las habilidades sociales, analizadas cualitativamente, muestran actitudes relativas a la mejora de estas áreas.

En conclusión, las TIC son herramientas creadas per se con una finalidad positiva; sin embargo, está en los usuarios la responsabilidad ética. El ámbito educativo las ha introducido, pero estas no han impactado correctamente, lo que lleva en ocasiones a utilizarlas para seguir haciendo lo mismo que se hacía con otras herramientas. No obstante, es preciso recordar que estos recursos permiten adecuar la práctica educativa a los diferentes ritmos de aprendizaje, compensar necesidades educativas, ofrecer estímulos que permiten captar, en mayor medida, la atención de los discentes y aumentar la autoestima y motivación de los estudiantes. De este modo, las TIC permiten una intervención con los estudiantes con TDAH de carácter multimodal y multidimensional.

\section{Referencias}

Alonso, J. A. (2020). La inclusividad de las TIC. Alumnos con TDAH. En E. Cobos, L. López, A. Molina, A.H. Jaén \& M. Padilla (Eds.), Claves para la innovación pedagógica ante los nuevos retos: respuestas en la vanguardia de la práctica educativa (pp. 3220-3226). Octaedro.

Álvarez Higuita, A. (2009). Caracterización de la escritura de textos narrativos mediada por un programa de reconocimiento de voz y un procesador de textos en niños con trastorno por déficit de atención con 
hiperactividad [Tesis doctoral]. Universidad de Antioquía, Medellín, Colombia. https://bibliotecadigital.udea.edu.co/handle/10495/1254

Brickenkamp, R., \& Cubero, N. S. (2002). d2, test de atención: Manual. TEA Ediciones.

Buela, G., Carretero, H., De los Santos, M., \& Bermúdez, M. P. (2005). MFF-20. Test de Emparejamiento de Figuras Conocidas 20. Adaptación española. $2^{\circ}$ edición. TEA Ediciones.

Campos, M. N., López, J. A., \& Marín, J. A. (2017). Funcionalidad de las TIC en el proceso de enseñanza y aprendizaje en niños diagnosticados con TDAH. En J. Ruiz-Palmero, J. Sánchez-Rodríguez, \& E. Sánchez-Rivas (Coords.), Innovación docente y uso de las TIC en educación. UMA Editorial.

Capodieci, A., Serafini, A., Dessuki, A., \& Cornoldi, C. (2019). Writing abilities and the role of working memory in children with symptoms of attention deficit and hyperactivity disorder. Child Neuropsychology, 25(1), 103-121. https://doi.org/10.1080/09297049.2018.1441390

Chousa, C., Martínez-Figueira, M. E., \& Raposo, M. (2017). Las TIC para la intervención educativa en TDAH: un estudio bibliométrico. Perspectiva educacional. Formación de profesores, 56(3), 142-161. https://doi.org/10.4151/07189729-Vol.56-Iss.3-Art.521

Crisol, E., \& Campos, M. N. (2019). Rehabilitación de las funciones ejecutivas en niños de 6 años con TDAH. Un estudio de caso. Revista de Currículum y Formación del Profesorado, 23(1), 285-306. https://doi.org/10.30827/profesorado.v23i1.9155

Deaño, M. (2007). DN-CAS Das Naglieri. Sistema de Evaluación cognitiva. Ediciones Gersam.

Doval, M. I., Raposo, M., \& Martínez-Figueira, M. E. (2013). As tecnoloxías da información e da comunicación na atención das NEAE. En M. E. Martínez-Figueira (Coord.), TIC para a inclusión de alumnado con necesidades específicas de apoio educativo (pp. 9-28). Universidade de Vigo.

Encalada, L. H. (2019). Herramientas TIC en la evaluación diagnóstica de alumnos con necesidades educativas especiales (Tesis doctoral). Universidad del Desarrollo, Santiago de Chile, Chile. https://repositorio.udd.cl/handle/11447/2831

Gil, J., \& Megías, A. (2017). Conocimientos sobre el trastorno por déficit de atención con hiperatividad (TDAH) en futuros profesionales del ámbito escolar. Bordón, revista de pedagogía, 69(3), 145-159. https://doi.org/10.13042/Bordon.2017.50278

González, G., \& Oliver, R. (2002). La informática en el DAH. Comunicación y Pedagogía, 182, 56-67.

López, J. A., Campos, M. N., Aznar, I., \& Rodríguez, C. (2020). Competencia digital del profesorado para la atención al alumnado con dificultades de aprendizaje. Una revisión teórica. REIFOP, 23(2). https://doi.org/10.6018/reifop.419171

Marco, M. (2010). Programas informáticos para trastornos de lectoescritura, Dislexia y/o TDAH. En P. Arnaiz, M. D. Hurtado, \& F. J. Soto (Coords.), 25 Años de Integración Escolar en España: Tecnología e Inclusión en el ámbito educativo, laboral y comunitario (pp. 1-8). Consejería de Educación, Formación y Empleo.

Rabadán, J. A., Parra, J., \& Hernández, E. (2017). Implementación y valoración de medidas educativas ordinarias adoptadas con el alumnado diagnosticado con trastorno por déficit de atención e hiperactividad en la comunidad autónoma de la Región de Murcia. Educación XX1, 20(1), 363-382. https://doi.org/10.5944/educxx1.17516 
Raposo, M., \& Salgado, A.B. (2015). Estudio sobre la intervención con Software educativo en un caso de TDAH. Revista nacional e internacional de educación inclusiva, 8(2), 121-138.

Rodillo, E. (2015). Trastorno por déficit de atención e hiperactividad (TDAH) en adolescentes. Revista Médica de Clínica Las Condes, 26(1), 52-59. https://doi.org/10.1016/j.rmclc.2015.02.005

\section{Cómo citar:}

Fernández-Menor, I. (2021). Evaluación de un estudiante con TDAH tras una intervención con recursos tecnológicos. Páginas de Educación, 14(2), 121-131.

https://doi.org/10.22235/pe.v14i2.2565

\section{Contribución autoral}

a) Concepción y diseño del trabajo; b) Adquisición de datos; c) Análisis e interpretación de datos; d) Redacción del manuscrito; e) revisión crítica del manuscrito.

I. F. M. ha contribuido en a, b, c, d, e.

\section{Editora científica responsable}

Mag. Florencia de León 